\title{
Erratum: Difference between two species of emu hides a test for lepton flavour violation
}

\author{
Christopher G. Lester and Benjamin H. Brunt \\ University of Cambridge, Department of Physics, Cavendish Laboratory, \\ JJ Thomson Avenue, Cambridge, CB3 OHE, U.K. \\ E-mail: lester@hep.phy.cam.ac.uk, brunt@hep.phy.cam.ac.uk
}

ERRATUM TO: JHEP03(2017)149

ABSTRACT: The paper named above should have used $m_{T}(e)+m_{T}(\mu)$ to illustrate its method, rather than $m_{T}(\mu)$, on account of the lack of first-order flavour symmetry of the latter.

\section{ARXIV EPRINT: 1612.02697}

The first three versions of this paper that were submitted to the arXiv [1-3] contain no fundamental mistakes needing correction. They used missing transverse momentum, $\boldsymbol{p}_{T}$, as an illustration of the type of variable that could be used to define the signal regions for which $e^{-} \mu^{+}$and $e^{+} \mu^{-}$yields would be compared. While the last of these versions was under review within JHEP, the authors made a decision, which they now regret, to change the illustrative variable from $\not p_{T}$ to the transverse mass of the muon, $m_{T}(\mu)$. This change may be seen in the fourth version of the paper [4], which is identical to that accepted for publication in JHEP in March 2017. Recently (July 2017) the authors realised that this last-minute change of variable introduced an inconsistency within the paper that was not present in the earlier $\boldsymbol{p}_{T}$-based versions. This erratum therefore provides:

1. evidence showing why $m_{T}(\mu)$ should not have replaced $\not p_{T}$, and why $m_{T}(e)+m_{T}(\mu)$ could instead have been used as an equally performant yet valid replacement, and

2. a set of figures that would replace those published in [4], if $m_{T}(e)+m_{T}(\mu)$ had been used instead of $m_{T}(\mu)$. These provide higher signal reach than $\not \boldsymbol{p}_{T}$ alone provides for the straw model considered, and almost identical reach to that reported (invalidly) for $m_{T}(\mu)$. 
Why was the change of variable described above invalid? The search method proposed in [4] looks for small differences between charges and flavours. It therefore requires a fiducial region that, at least to first order, is blind to exchange of $e$ and $\mu$ flavours. The proofs of the charge-flavour 'conspiracy' (equation (2.1) of [4]) in appendix A of [4] also require this. A signal region that requires $\not_{T}$ to exceed a fixed threshold meets this flavourblind requirement if electrons and muons have equal acceptance, but one built from a transverse mass constructed exclusively from a muon does not, as the example given below shows. Accordingly, while $\not p_{T}$ was a good choice of variable for which the charge-flavour 'conspiracy' holds, $m_{T}(\mu)$ is a variable for which that 'conspiracy' can be (and in fact for di-boson production processes is) violated at a low level as described below.

Example. Here we give a hypothetical example that illustrates the basic mechanism by which di-boson events can cause $m_{T}(\mu)$ to violate the charge-flavour 'conspiracy' at a low level, even though the underlying physics of di-boson events is charge-flavour symmetric.

Suppose that proton-proton collisions produce events with two leptons of opposite charge but with all flavour combinations $\left(e^{+} \mu^{-}, e^{+} e^{-}, \mu^{+} \mu^{-}\right.$and $\left.\mu^{+} e^{-}\right)$being equally likely. In such a scenario it could still be the case that positively charged leptons, of either flavour, might tend to be more 'forward"1 than the negatively charged leptons. This might happen due to quark-gluon or quark-antiquark processes through which the positively charged lepton in the final state preferentially inherits longitudinal momentum from valence quarks in the proton, rather than softer momentum from gluons or sea (anti)quarks. In such a scenario, a signal region requiring 'that a muon or antimuon should be far forward' would select more events of type $e^{-} \mu^{+}$than of type $e^{+} \mu^{-}$, since positive muons tend to be more forward than negative muons. If the signal region of the previous example were replaced with one demanding 'a forward lepton of either flavour' then the first-order bias of the type described would disappear. ${ }^{2}$

The actual size of the undesired first-order bias from di-boson events was small but non-negligible. Evidence of this could be seen in figure 1 of [4] above $m_{T}(\mu)$ of $450 \mathrm{GeV}$, where the expected number of $W W$ events (cyan) was seen to be around 5 or 6 in the $e^{+} \mu^{-}$ channel, but to be closer to 2 in the $e^{-} \mu^{+}$channel. Other backgrounds contributed around 25 events per charge in the same regions. This di-boson process thus generated a small 'wrong way' (i.e. non-conspiratorial) bias, on account of the invalidity of $m_{T}(\mu)$ as a search variable. The smallness of this bias would not have changed the conclusion of the $20 \mathrm{fb}^{-1}$ illustration given in [4], but clearly would have become an issue given sufficient data.

The correction. The (regretted) last-minute decision to move from $\not p_{T}$ to $m_{T}(\mu)$ was motivated by the better signal reach that resulted from this change. Though that choice of variable was invalid, reach improvements of similar size could have easily been made using any of a large number of first-order flavour-blind replacements for $m_{T}(\mu)$. The simplest of

\footnotetext{
${ }^{1}$ 'Forward' here means having a pseudorapidity of large magnitude.

${ }^{2}$ No signal region is ever entirely flavour-blind. Electrons and muons are reconstructed separately, so there will always be differences in energy scale or acceptance that will not be fully modelled. These differences will only show up at second-order if the core signal region definition treats all flavours equally. The need to evaluate the size of remaining (second-order) biases is never avoided entirely.
} 


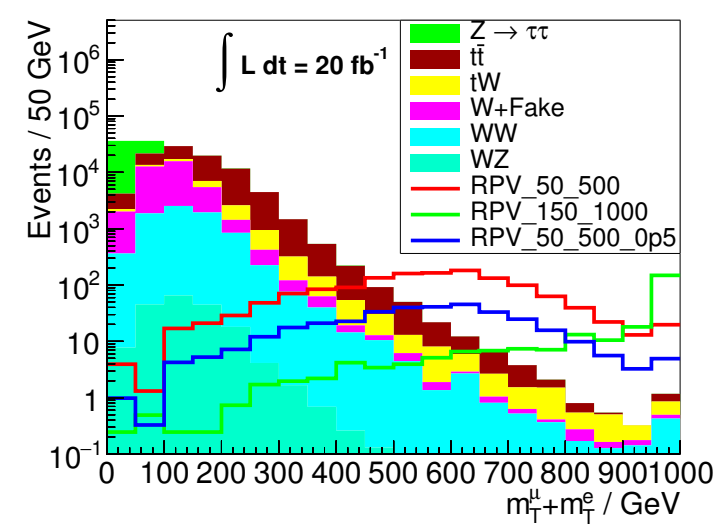

(a) $m_{T}(e)+m_{T}(\mu)$ in $e^{+} \mu^{-}$events.

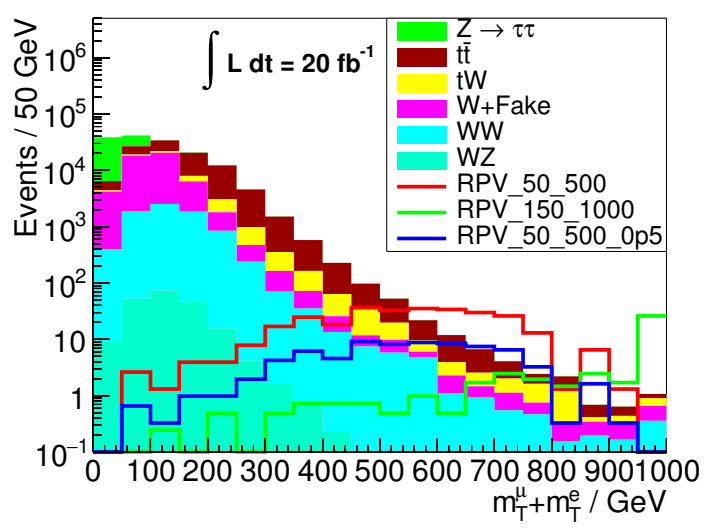

(b) $m_{T}(e)+m_{T}(\mu)$ in $e^{-} \mu^{+}$events.

Figure 1. The expected distributions of $m_{T}(e)+m_{T}(\mu)$ in events with OSDF leptons $\left(e^{+} \mu^{-}\right.$and $e^{-} \mu^{+}$in (a) and (b) respectively).

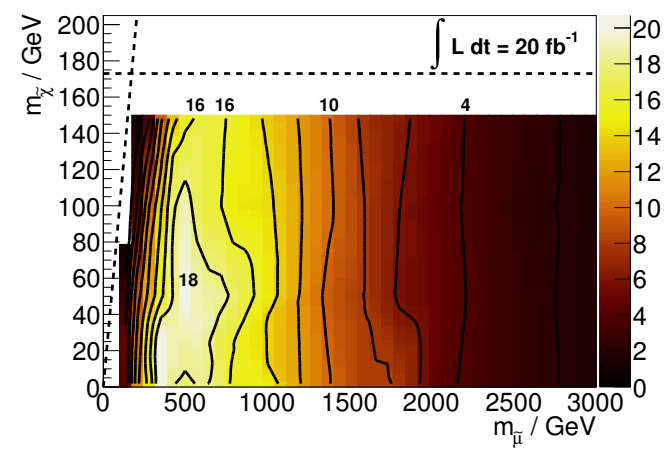

Figure 2. The median value of $f$ (in effect the expected sensitivity of the method) for the grid of $\lambda_{231}^{\prime}=1$ signal models. Contour lines show integer values of sensitivity.

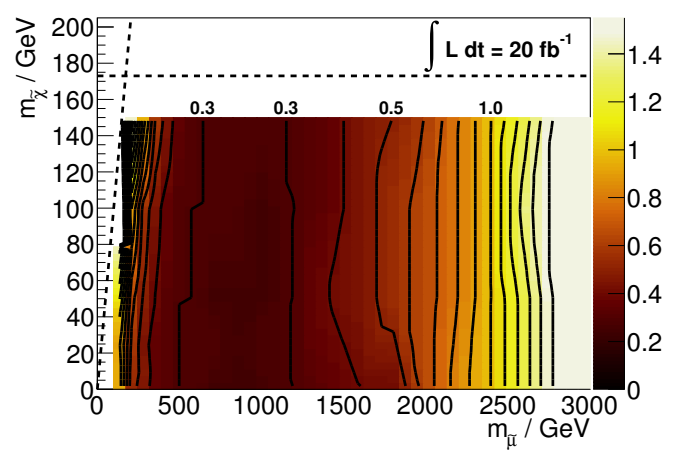

Figure 3. The minimal value in each model of the $\lambda_{231}^{\prime}$ coupling for which a sensitivity of $f \geq 3$ is achieved. Contour lines are drawn at intervals of 0.1 .

these is $m_{T}(e)+m_{T}(\mu)$. Figures 1, 2 and 3 herein are, respectively, replacements for figures 1, 5 and 6 of [4]. They show the signal distributions, background distributions, and signal sensitivities that would have been seen if $m_{T}(e)+m_{T}(\mu)$ had replaced all occurrences of $m_{T}(\mu)$ in [4]. Figure 2 shows that, following the replacement of the invalid variable with its more correct flavour-blind counterpart, the 2-sigma reach in slepton mass remains at around $\sim 2800 \mathrm{GeV}$, matching the equivalent contour in [4].

Open Access. This article is distributed under the terms of the Creative Commons Attribution License (CC-BY 4.0), which permits any use, distribution and reproduction in any medium, provided the original author(s) and source are credited.

\section{References}

[1] C.G. Lester and B.H. Brunt, Difference between two species of e $\mu$ hides a test for lepton flavour violation, arXiv: $1612.02697 \mathrm{v} 1$. 
[2] C.G. Lester and B.H. Brunt, Difference between two species of ep hides a test for lepton flavour violation, arXiv: $1612.02697 \mathrm{v} 2$.

[3] C.G. Lester and B.H. Brunt, Difference between two species of ep hides a test for lepton flavour violation, arXiv:1612.02697v3.

[4] C.G. Lester and B.H. Brunt, Difference between two species of ep hides a test for lepton flavour violation, arXiv: $1612.02697 \mathrm{v}$. 